\title{
Medizinische Hilfe für die Ärmsten
}

\author{
Urban Pachlatko
}

Von Ende Februar bis Mitte April hatte ich die Möglichkeit, mit einer deutschen Hilfsorganisation in Dhaka der Hauptstadt von Bangladesch - einen sechswöchigen medizinischen Einsatz zu machen.

Die German Doctors, wie die Organisation heisst, arbeiten weltweit in verschiedenen Armutsgebieten und versuchen dort für die Ärmsten eine gewisse Basismedizin zu betreiben. Es sind Deutsch sprechende Ärzte, die jeweils für etwa sechs Wochen im Einsatz sind.

In Dhaka wohnen wir in einem Haus, in dem, neben einer Klinik, auch eine Schule für «Slumkinder» untergebracht ist. Etwa 600 Kinder, in zwei Gruppen hintereinander, erhalten dort eine Ausbildung und eine warme Mahlzeit pro Tag. Wir fahren vormittags mit einem Minibus in unterschiedliche Slums und arme Wohngebiete, nachmittags arbeiten wir in einer Ambulanz in dem Haus, wo wir auch wohnen. Wir kümmern uns sozusagen um die hausärztliche Versorgung der Ärmsten, die sich das Geld für einen Arzt- oder Krankenhausbesuch nicht leisten können. Wir, das sind zwei deutsch sprechende Ärzte und ein Team aus Einheimischen, die für uns übersetzen, die Patienten aufnehmen, sich um die Medikamente kümmern und uns hin- und herfahren.

Uns begegnen sehr oft die gleichen Beschwerden, die man auch hier in einer Hausarztpraxis findet, nämlich
Husten, Erkältungen, Fieber, Rückenschmerzen, verschiedenste Verdauungsbeschwerden und «all over body pain", was eher als ein allgemeines Unwohlsein zu verstehen ist. Viele der Beschwerden sind auch Folgen der oft harten körperlichen Arbeiten, denen die Slumbewohner nachgehen. Dass die Rikschafahrer und die Leute, die mit Hämmern Backsteine zerkleinern, an Überlastungsschmerzen leiden, wundert keinen, aber wirksame Therapien dagegen stehen nicht zur Verfügung. Eine vorübergehende Arbeitsunfähigkeit bleibt Wunschdenken. Die sozialen Probleme kommen dann gerade bei den Armen noch zur gesundheitlichen Situation dazu und lassen die Lage oft eher hoffnungslos aussehen. Trotz allem sind die Menschen nicht unglücklicher als bei uns und tragen ihr Schicksal oft auf bewundernswerte Art.

Ein paar Einblicke in Patientengeschichten:

- Alte Frau, die nicht mehr arbeiten kann (75-jährig). Schwäche und Appetitlosigkeit, Magenschmerzen (Hunger?) und verschiedene Gelenkschmerzen sind ihr heutiges Leiden. Bei weiterem Nachfragen kommt heraus, dass sie nun bei ihrer Tochter lebt, die aber selber eher am Existenzminimum ist. Die Tochter ist als Textilarbeiterin tätig, das heisst, sie muss den ganzen Tag nähen oder zuschneiden oder bügeln oder ... Sie verdient zwar genug für sich und vielleicht ein Kind, aber

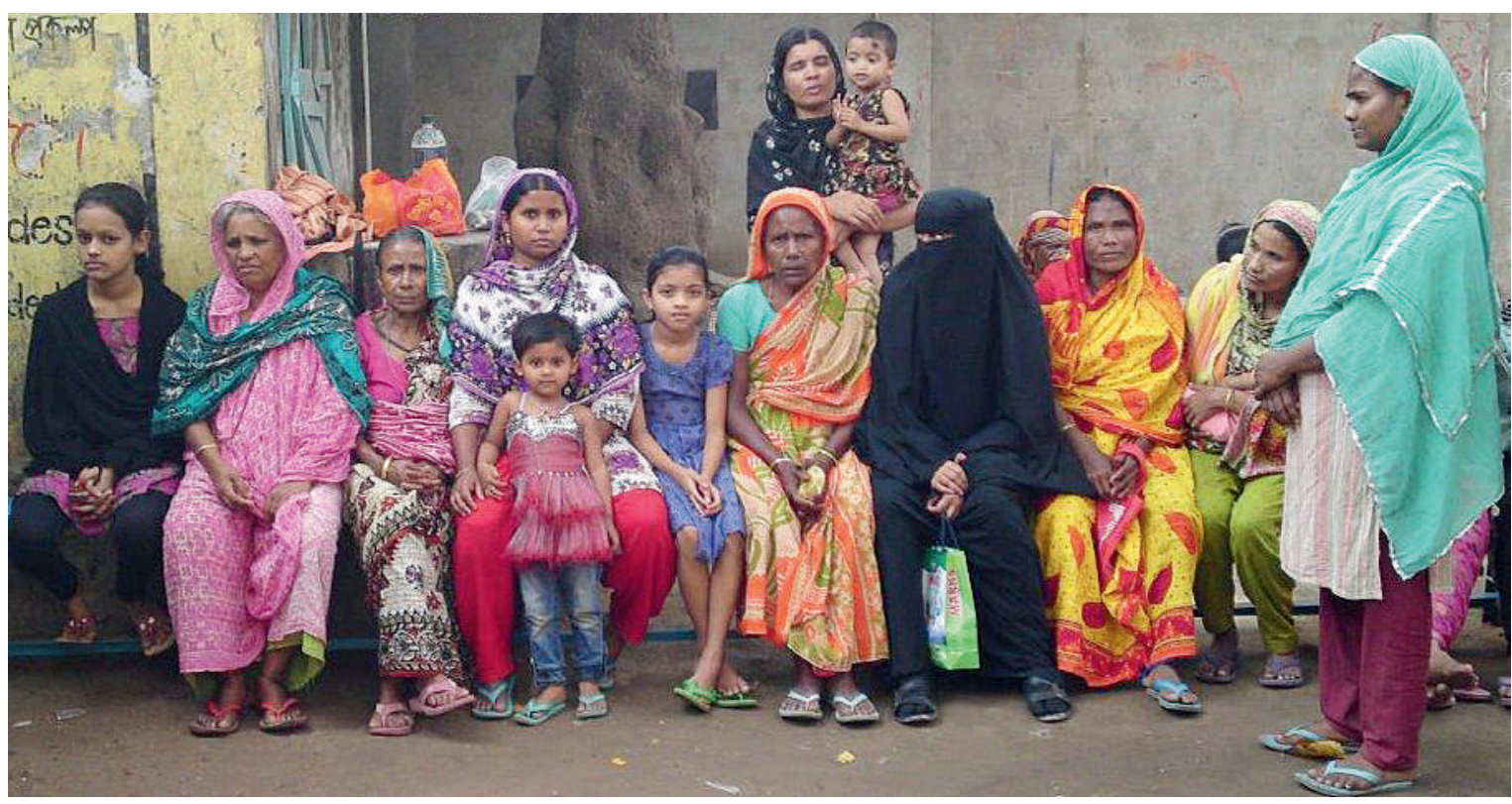

Das «Wartezimmer». 


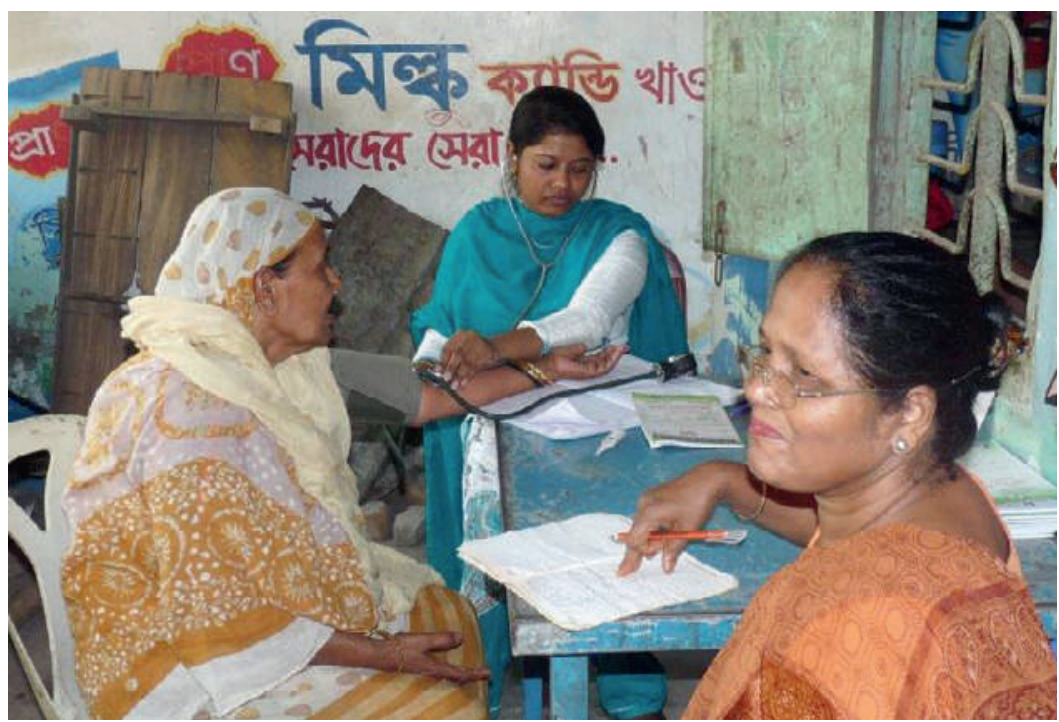

Eine Patientin der German Doctors wird aufgenommen.

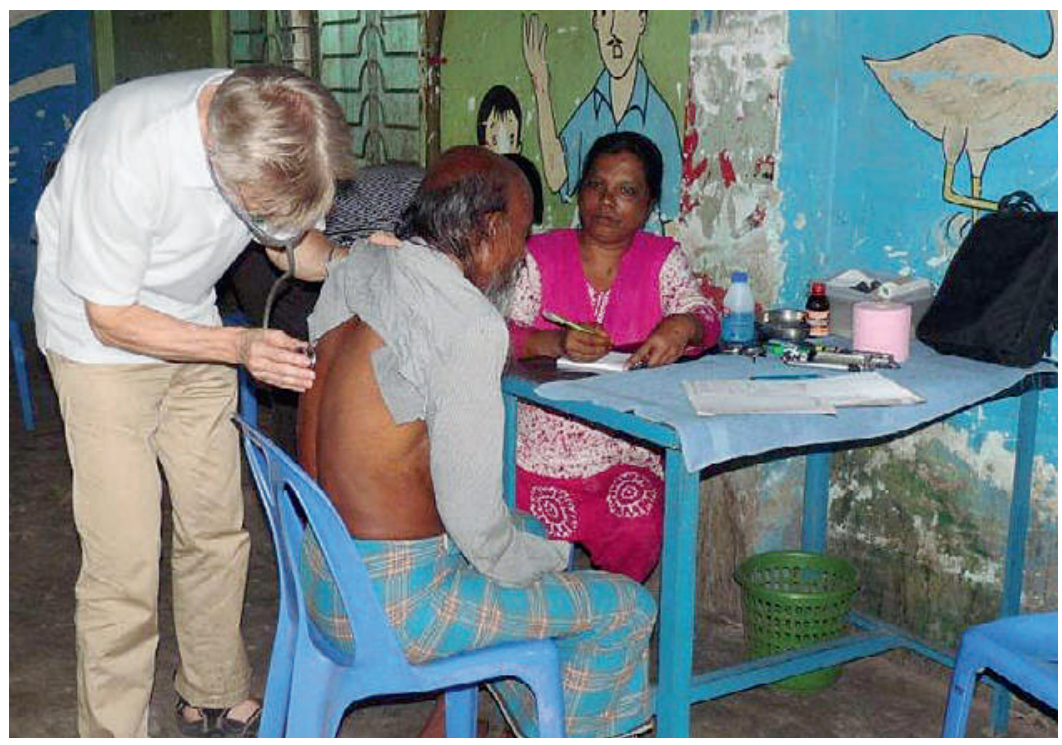

Oft haben Patienten ganz ähnliche Beschwerden wie bei uns.

für die Mutter reicht es einfach nicht mehr. So sagt die Mutter einfach, sie habe keinen Appetit, damit sie den andern nichts wegisst, selber aber verhungert sie langsam ...

- Mann, alt, beidseitige Hüftarthrose, ist unstabil auf den Beinen und fällt oft hin. Heute kommt er mit einer Verletzung des rechten Knies, einer offenen Wunde, weil er vor ein paar Tagen wieder einmal gefallen ist. Durch Schmerzen geschwächt, dadurch kaum mehr erwerbstätig, dreht sich die Armutsspirale weiter. Er wird schwächer werden, noch häufiger fallen, bis er dann nicht mehr aufstehen wird ...

Dr. med. Urban Pachlatko Leeacherstrasse 21 CH-8123 Ebmatingen pachlatko[at]ggaweb.ch
Tochter selber hatte einen Schlaganfall und nun eine Fazialisparese, das heisst, die Gesichtsmuskeln sind auf einer Seite gelähmt. Die Tochter muss neben ihrer Mutter auch noch für ihre eigene Tochter schauen, da ihr Mann drogenabhängig ist und alles, was er mit dem Rikschafahren verdient, sofort für seine Sucht verbraucht.

- Ein persönliches «Highlight» für mich war eine alte Frau, die vier Tage vorher bei einem Sturz ihren Vorderarm an typischer Stelle gebrochen hatte. Wir machten eine lokale Anästhesie in den Bruchspalt und gipsten dann den reponierten Arm mit «richtigen» Gipsbinden ein, und das ohne Röntgen, einfach nach Gefühl! Mir kam zugute, dass ich in Valbella vor 30 Jahren viele Radiusfrakturen reponieren und gipsen musste. Es war wie ein Lächeln von Gott, dass Er das, was ich gelernt habe, auch hier wieder gebrauchen konnte. Die Frau kam dann am nächsten Tag zur Gipskontrolle und war begeistert, dass sie keine Schmerzen mehr hatte.

Die Regierung hat in den letzten Jahren mehrere Spitäler aufgemacht, die auch die Ärmsten behandeln, und so können wir in komplizierteren Fällen die Leute auch dorthin schicken. Es gibt lange Wartezeiten, aber wenn wir die Patienten gezielt zuweisen, erhalten sie eine recht gute Diagnostik. Medikamente bekommen sie dann aber meist nur für einen Tag und dazu ein Rezept in die Hand gedrückt, mit dem sie ihre weitere Medikation kaufen sollten. Auch wenn Medikamente nicht sonderlich teuer sind und praktisch alle im Land selber hergestellt werden, haben die Ärmsten oft nicht genug Geld, um sich eine längere Behandlung zu leisten, und hören dann einfach damit auf.

Die Behandlung der Tuberkulose ist hier eine Ausnahme, da erhalten die Patienten regelmässig ihre Medikation, und es wird auch gut kontrolliert. Für unsere Therapien haben wir ein beschränktes, der Situation gut angepasstes Medikamentensortiment, das wir den Patienten fast gratis abgeben. Diese Arbeit, die ich in Dhaka machen durfte, hat mir grosse Freude gebracht. Die Patienten sind dankbar, dass jemand sie wirklich untersucht und ihnen zum Teil einfach auch nur mit einem offenen Ohr begegnet. Manchmal fühlte ich mich aber auch so hilflos, wenn das eigentliche Übel gar nicht angegangen werden kann, da wir ja fast «nur» medizinisch helfen können. Die Armut, die kaum vorhandenen staatlichen sozialen Strukturen und der schiere Bevölkerungsdruck lasten auf dem Buckel und der Gesundheit ebendieser Bevölkerung. In Anbetracht der 160 Millionen Einwohner erscheint der Einsatz wirklich nur als Tropfen auf einen sehr heissen Stein, aber ich denke, dass die paar hundert Menschen, denen wir helfen konnten, trotzdem dankbar sind. 